\title{
A Prospective Study Comparing the Results of Endoscope Assisted Versus Microscope Assisted Myringoplasty
}

\author{
Aftab Ahmed*, Shamsheer Alam, Shahab Farkhund Hashmi and Syed Abrar Hasan \\ Department of Otorhinolaryngology, Aligarh Muslim University, India
}

Submission: June 26, 2016; Published: July 14, 2016

*Corresponding author: Aftab Ahmed, Senior Resident, Department of Otorhinolaryngology, J.N. Medical College, Aligarh Muslim University, Aligarh, Pin-202002, India, Tel: +918791520112; Email: aftab2k3@gmail.com

\begin{abstract}
Objective: To assess the operative time, post operative pain, graft uptake and audiological gain in the group of the patients undergoing the endoscope assisted and the microscope assisted myringoplasty.

Setting: Tertiary referral hospital.

Subjects: The study was prospective in nature and comprised of 100 patients suffering from the chronic otitis media inactive mucosal disease. Patients attending the Otorhinolaryngology out patients department were included in this study.

Methods: Transcanal myringoplasty or type-1 tympanoplasty was performed by either the endoscope or the microscope assistance. Endaural/Postaural approach was undertaken in few microscope assisted myringoplasty. Underlay technique of myringoplasty was performed using temporalis fascia graft in all the patients suffering from the inactive mucosal chronic otitis media. Post operative evaluation was done in terms of operative time, pain, graft uptake and audiological gain. The patients were followed up for a period of 6 months.

Results: The operative time in the patients undergoing the endoscope assisted myringoplasty was $49.76 \pm 3.18$ minutes whereas it was $62.37 \pm 3.69$ minutes in the patients undergoing the microscope assisted Transcanal myringoplasty and $72.15 \pm 2.56$ minutes in the patients undergoing the microscope assisted Endaural/Postaural myringoplasty. Post operative pain was assessed the next day after the surgery, it was less severe in the Transcanal approach endoscope/microscope assisted myringoplasty groups and more severe in the microscope assisted Endaural/Postaural approach myringoplasty group. The difference between the groups was statistically significant. The tympanic membrane graft uptake and audiological gain was assessed at 1 month, 3 months and 6 months post operatively. The successful closure of the tympanic membrane perforation was higher with the endoscope assisted myringoplasty as compared to the microscope assisted myringoplasty at 1 month, 3 months and 6 months of follow up but the difference was not statistically significant. The mean audiological gain following myringoplasty with both the techniques used was noted. The mean audiological gain was found to be marginally better with the endoscope assisted myringoplasty at follow up of 1, 3 and 6 months as compared to the microscope assisted myringoplasty, however this difference was not statistically significant.

Conclusion: The operative time was less in the endoscope assisted technique as compared to the microscope assisted technique. The post operative morbidity (evaluated in terms of post operative pain) was less in the group of patients undergoing the Transcanal endoscope assisted as compared to microscope assisted myringoplasty. The post operative pain was more severe in the Endaural/Postaural approach than the Transcanal approach microscope assisted myringoplasty. There was no statistically significant difference in either graft uptake or post-operative audiological gain in the patients undergoing myringoplasty by endoscope assisted and microscope assisted technique. The endoscope assisted myringoplasty has advantages of better assessment of ossicular chain and in the patients with canal overhang it improves the visualisation of the margins of the perforation thus obviating the need for canalplasty. Thus the endoscope assisted myringoplasty can be used as a preferred alternative to the conventional microscope assisted myringoplasty, with better post operative results and reduced operating time and morbidity of the surgical procedure.
\end{abstract}

Keywords: Chronic otitis media (COM); Myringoplasty; Endoscope assisted myringoplasty; Microscope assisted myringoplasty; Temporalis fascia graft

\section{Introduction}

Chronic otitis media has been an important middle ear disease since pre historic times. Its incidence appears to depend to some extent on race and socioeconomic factors. Poor living con ditions, overcrowding, poor hygiene and malnutrition have been suggested as a basis for the wide spread prevalence of Chronic otitis media (COM) in developing countries. Histopathologically COM is an inflammatory process of the mucoperiosteal lining of the 
middle ear cleft. The middle ear mucosa is edematous, with submucosal fibrosis and infiltration by chronic inflammatory cells. The mucosal edema can lead to the formation of an aural polyp, which can project into the external auditory canal. Bone erosion including ossicular resorption, mucosal ulceration and formation of granulation tissue is the correlate of persistent otorrhea. Mucosal thickening can impair ossicular and tympanic membrane mobility, aggravated by the deposition of the hyaline material i.e. tympanosclerosis [1].

Various methods have been used for reparative procedure of the tympanic membrane perforation (Myringoplasty) [2,3]. Perforation of tympanic membrane and hearing loss of conductive type are indications of myringoplasty [4]. The closure of the tympanic membrane perforation by myringoplasty is one of the most common surgical procedures performed in Otorhinolaryngology practice. Resident training in otology often begins with the myringoplasty procedures with temporalis fascia graft as the usual graft material, traditionally performed and taught using operating microscope, as with the most otological procedures.

Endoscopy has increasing role to play in the ear surgery, namely photo documentation of tympanic membrane [5], cholesteatoma surgery [6], surgery of the facial nerve [7], and anatomy of the sinus tympani [8]. Endoscope assistance has improved the visual exposure of hidden and deep structures, it provides a wide angle view of the tympanic membrane remnant and middle ear structures. Endoscope brings surgeon's eye to the tip of the endoscope thus magnifying the image by just getting close to the structures. It can be easily negotiated through tortuous EAC, thus obviating the need for canalplasty. Despite the increasing number of publications on the role of the endoscopy in the management of otitis media surgery, there has been a scarcity of publications on endoscopic myringoplasty [9], with most relying on the graft placement without tympanomeatal flap elevation in small to medium perforations. There have been very few studies involving direct comparison between endoscope assisted and microscope assisted myringoplasty using flap elevation technique [10].

The effects of incision type in tympanoplasty on the cosmesis [11] and the presence of bony canal overhangs necessitating canalplasty [12] have prompted the use of exclusive endoscopic Transcanal myringoplasty in the management of tympanic membrane perforations of different sizes and locations. The current study was undertaken to compare the microscope assisted and the endoscope assisted methods of myringoplasty/type- 1 tympanoplasty directly using temporalis fascia by underlay placement method. The objectives of this study were:

1. To assess operative time in both the procedures.

2. To assess morbidity index i.e. pain level after surgery in both the procedures.

3. To assess graft uptake in both the procedures.

4. To assess hearing status in both the procedures.

\section{Materials and Methods}

The study comprised of 100 patients of COM, inactive mucosal disease of both the sexes and age group 15-50 year. Group A consisted of 50 patients who underwent endoscope assisted myringoplasty and Group B also consist of 50 patients who underwent microscope assisted myringoplasty. In the endoscope assisted group Transcanal approach of myringoplasty was performed in all patients. In microscope assisted group 30 patients underwent Transcanal approach myringoplasty and 20 patients underwent Endaural/Postaural approach myringoplasty. The graft material used in all the procedures was temporalis fascia. The patients were subjected to detailed history, general, systemic and local examination which included clinical examination of ear, nose, paranasal sinuses, larynx and pharynx. Patients with obvious ossicular dysfunction and external ear pathology were excluded from the study. Hearing impairment was assessed by pure tone audiometry with or without masking. The available four frequency (i.e. 0.5,1,2 and $4 \mathrm{kHz}$ ), preoperative air bone gap was recorded for each case.

\section{Technique of microscope assisted myringoplasty}

The surgery was performed under local anaesthesia with all the three approaches postaural, endaural and Transcanal whichever allowed better exposure. The temporalis fascia graft was harvested; the margins of the perforation were freshened using operating microscope. The tympanomeatal flap was then elevated and ossicular chain inspected. The temporalis fascia graft was placed in such a manner that it extends under the margin of perforation and a small part should also extend over the posterior canal wall. Tympanomeatal flap was then reposited back and abgel placed in the ear canal. Post operative antibiotics, anti inflammatory, nasal decongestant and tear drops were used.

\section{Technique of endoscope assisted myringoplasty}

In the endoscopy group, zero and thirty degree $2.5 \mathrm{~mm}$ rigid endoscopes were used and the procedure was performed using an exclusive Transcanal approach. Temporalis fascia graft was harvested from a separate supra-auricular hairline incision. The endoscope was held in the left hand and instrument in the right hand, no endoscope holder was used. After debridement of margins, the tympanomeatal flap was elevated depending upon the size and location of the perforation. The middle ear cavity was inspected and ossicular chain integrity ensured. Temporalis fascia graft was placed and tympanomeatal flap repositioned and ear canal was packed with abgel. Post operative medications were same as in microscope assisted group.

The operative time of the each surgery was recorded. The time taken to perform the surgery was calculated from the starting of the graft harvesting till the closure of the surgery and dressing of wound. The patients were followed up one day post operatively for pain and at 1 month, 3 months and 6 months for tympanic membrane graft uptake and audiological gain. For evaluation of the post operative pain the patients were interviewed the next day of surgery and they were asked to grade the post operative pain on the numerical rating scale, 0 -10. Each patient was assigned a 
definite number on the 0-10 scale, depending upon the severity of the post operative pain. Mean score was calculated for each group by dividing the sum of the score in that group by the total number of cases in the same group. The groups were compared for statistically significant difference in pain. Successful graft uptake, on otoscopic examination was taken only in the cases in which no remnant of perforation was present in any of the quadrant of tympanic membrane. Audiological gain was calculated by subtracting post operative air-bone conduction gap from pre operative airbone conduction gap. Mean audiological gain was calculated for each group by dividing the sum of the audiological gain in that group by the total number of cases in the group.

\section{Inclusion criteria}

1. Age group 15 - 50 years.

2. Chronic otitis media mucosal variety, inactive for at least 6 weeks.

3. Intact middle ear conducting mechanism.

4. Adequate cochlear reserve.

\section{Exclusion criteria}

1 . Age $<15$ years and $>50$ years.

2. Patients having tympanosclerosis, ossicular chain disorder, presence of cholesteatoma and retraction pocket.

3. Patients with sensorineural deafness.

4. Patients with complications of chronic otitis media.

5. Patients having sinonasal pathology like chronic sinusitis, allergic rhinitis etc.

6. Patients having any systemic illness like diabetes mellitus, hypertension etc.

\section{Data analysis}

Data was analyzed by using SPSS 20. Chi- square test was used for comparing graft uptake and unpaired t-test was used for statistical comparison of post operative audiological gain. One way analysis of variance (ANOVA) test was used for statistical comparison of operative time and post operative pain. Tukey's Honest Significant Difference post hoc test was used after ANOVA. P value of $<0.05$ was considered significant while $p$ value $<0.01$ was considered highly significant.

\section{Results}

The present study was carried out for a period of one year from June 2015 to May 2016. The study included 100 patients who underwent Myringoplasty/type-1 Tympanoplasty for chronic otitis media (inactive mucosal type). In our study of 100 patients 43 were male and 57 were female with a male to female ratio of 1:1.3. The age of the patient ranged 15-50 year. In our study of 100 patients of COM (inactive mucosal disease), 34 patients had small size $(<50 \%), 51$ patients had medium size (50-75\%) and 15 patients had large size perforation $(>75 \%)$. Out of 100 patients, 24 patients were having anterior perforation, 38 patients were having central perforation, and 28 patients were having posterior perforation of the pars tensa. As previously mentioned the patients with obvious ossicular dysfunction and external ear pathology were excluded from the study (Table 1) is summarizing the cases on the basis of demographic variables.

Table 1: Classification of cases on the basis of demographic variables.

\begin{tabular}{|c|c|c|}
\hline & No. of Cases & Percentage \\
\hline 1. Age Group (Years) & & \\
\hline $15-25$ & 54 & $54.0 \%$ \\
\hline $26-35$ & 31 & $31.0 \%$ \\
\hline $36-50$ & 15 & $15.0 \%$ \\
\hline 2. Sex & & \\
\hline Male & 43 & $43.0 \%$ \\
\hline Female & 57 & $57.0 \%$ \\
\hline 3. Population & & \\
\hline Rural & 61 & $61.0 \%$ \\
\hline Urban & 39 & $39.0 \%$ \\
\hline
\end{tabular}

\section{Operative Time}

The time to perform the surgery was compared between two groups of the patients. The time taken to perform the surgery was calculated from the starting of the graft harvesting till the closure of the surgery and dressing of wound. In the Transcanal endoscope assisted surgery it was $49.76 \pm 3.18$ minutes whereas in the Transcanal microscope assisted group it was $62.37 \pm 3.69 \mathrm{~min}-$ utes and Endaural/Postaural microscope assisted group it was $72.15 \pm 2.56$ minutes. The average time taken in the microscope assisted myringoplasty was greater than the endoscopic assisted procedure. Moreover the time taken in performing the surgery by Endaural/Postaural approach was more than the Transcanal approach in the microscope assisted group. The results of operative time are summarized in (Table 2).

Table 2: Comparison of operative time for different techniques used.

\begin{tabular}{|c|c|c|c|c|}
\hline Technique & Cases & $\begin{array}{l}\text { Mean opera- } \\
\text { tive time }\end{array}$ & $\begin{array}{l}\text { SD } \\
( \pm)\end{array}$ & P-value \\
\hline $\begin{array}{c}\text { Transcanal microscope } \\
\text { assisted }\end{array}$ & 50 & 49.76 & 3.18 & \multirow{3}{*}{$\mathrm{P}<0.001$} \\
\hline $\begin{array}{l}\text { Transcanal endoscope } \\
\text { assisted }\end{array}$ & 30 & 62.37 & 3.96 & \\
\hline $\begin{array}{l}\text { Endaural / Postaural } \\
\text { microscope assisted }\end{array}$ & 20 & 72.15 & 2.56 & \\
\hline
\end{tabular}

\section{Post operative morbidity}

The post operative morbidity of the surgery was greater in the group of the patients undergoing microscope assisted myringoplasty by Endaural/Postaural approach; this was evaluated in terms of post operative pain. The patients undergoing Transcanal approach myringoplasty by both the endoscope assisted and microscope assisted techniques have less severe post operative pain as compared to the patients undergoing myringoplasty by Endaural/Postaural approach. The results of post operative pain are summarized in (Table 3). 


\section{Global Journal of Otolaryngology}

Table 3: Comparison of post operative pain for different techniques used.

\begin{tabular}{|c|c|c|c|c|}
\hline Technique & Cases & Mean Score & SD ( \pm ) & P- value \\
\hline $\begin{array}{c}\text { Transcanal microscope } \\
\text { assisted }\end{array}$ & 50 & 3.56 & 1.28 & \\
\cline { 1 - 3 } $\begin{array}{c}\text { Transcanal endoscope } \\
\text { assisted }\end{array}$ & 30 & 4.40 & 1.35 & P $<0.001$ \\
\cline { 1 - 3 } $\begin{array}{c}\text { Endaural / Postaural } \\
\text { microscope assisted }\end{array}$ & 20 & 5.40 & 1.54 & \\
\cline { 1 - 3 }
\end{tabular}

\section{Post operative tympanic membrane graft uptake}

Evaluation of the tympanic membrane graft uptake following myringoplasty was done at 1 month, 3 months and 6 months post operatively. On otoscopic examination, successful uptake of the tympanic membrane graft was taken in the cases in which no remnant of perforation was present in any of the quadrant. The success rate of graft uptake for the endoscope assisted myringoplasty was $88.0 \%$ and for the microscope assisted group was $84.0 \%$ at one month post operatively. Out of 100 patients, 94 patients attended follow up after 3 months of surgery whereas only 85 patients were available for the follow up at 6 months. Others patients did not return for follow up. The tympanic membrane graft uptake at 1 month, 3 months and 6 months after the surgery for different techniques used are summarized in (Table 4).

Table 4: Table showing graft uptake for different techniques used.

\begin{tabular}{|c|c|c|c|c|}
\hline & $\begin{array}{l}\text { Successful } \\
\text { closure }\end{array}$ & $\begin{array}{l}\text { Failure of } \\
\text { closure }\end{array}$ & $\begin{array}{c}\text { Success } \\
\%\end{array}$ & P-value \\
\hline \multicolumn{5}{|l|}{ At one month } \\
\hline Endoscope assisted & 44 & 6 & $88.0 \%$ & \multirow{3}{*}{$\mathrm{P}=0.56$} \\
\hline $\begin{array}{l}\text { Microscope as- } \\
\text { sisted }\end{array}$ & 42 & 8 & $84.0 \%$ & \\
\hline Total & 86 & 14 & $86.0 \%$ & \\
\hline \multicolumn{5}{|l|}{ At three months } \\
\hline Endoscope assisted & 40 & 6 & $86.9 \%$ & \multirow{3}{*}{$P=0.62$} \\
\hline $\begin{array}{l}\text { Microscope as- } \\
\text { sisted }\end{array}$ & 40 & 8 & $83.3 \%$ & \\
\hline Total & 80 & 14 & $85.1 \%$ & \\
\hline \multicolumn{5}{|l|}{ At six months } \\
\hline Endoscope assisted & 37 & 5 & $88.1 \%$ & \multirow{3}{*}{$P=0.39$} \\
\hline $\begin{array}{l}\text { Microscope as- } \\
\text { sisted }\end{array}$ & 35 & 8 & $81.4 \%$ & \\
\hline Total & 72 & 13 & $84.7 \%$ & \\
\hline
\end{tabular}

\section{Post operative audiological gain}

In our study pure tone audiometry was done at 1 month, 3 months and 6 months postoperatively and audiological gain was evaluated. AB gap (air -bone conduction gap) per case was calculated as mean of $A B$ gap at four frequencies $(0.5,1,2 \& 4 \mathrm{KHz})$ pre operatively and post operatively. Audiological gain was calculated for each patient by subtracting the post op $A B$ gap from the pre op AB gap. The mean was calculated for each variable by dividing the sum of audiological gain in that group by the total number of cases in the same group. In our study of 100 patients only 94 pa- tients attended the follow up after 3 months of surgery whereas 85 patients were available for the follow up at 6 months, others were lost for follow up. Others patients did not return for follow up. Mean audiological gain at 1 month, 3 months and 6 months after the surgery for different techniques used are summarized in (Table 5).

Table 5: Table showing audiological gain for different techniques used.

\begin{tabular}{|c|c|c|c|c|c|}
\hline & $\begin{array}{l}\text { Graft mate- } \\
\text { rial }\end{array}$ & Cases & $\begin{array}{l}\text { Mean Au- } \\
\text { diological } \\
\text { gain }\end{array}$ & $\begin{array}{l}\text { SD } \\
( \pm)\end{array}$ & P-value \\
\hline \multirow{2}{*}{$\begin{array}{l}\text { At one } \\
\text { month }\end{array}$} & $\begin{array}{c}\text { Endoscope } \\
\text { assisted }\end{array}$ & 50 & 9.33 & 3.19 & \multirow{2}{*}{$\mathrm{P}=0.45$} \\
\hline & $\begin{array}{l}\text { Microscope } \\
\text { assisted }\end{array}$ & 50 & 8.83 & 3.47 & \\
\hline \multirow{2}{*}{$\begin{array}{l}\text { At three } \\
\text { months }\end{array}$} & $\begin{array}{c}\text { Endoscope } \\
\text { assisted }\end{array}$ & 46 & 9.53 & 3.04 & \multirow{2}{*}{$\mathrm{P}=0.36$} \\
\hline & $\begin{array}{l}\text { Microscope } \\
\text { assisted }\end{array}$ & 48 & 8.93 & 3.38 & \\
\hline \multirow{2}{*}{$\begin{array}{c}\text { At six } \\
\text { months }\end{array}$} & $\begin{array}{c}\text { Endoscope } \\
\text { assisted }\end{array}$ & 42 & 9.75 & 2.97 & \multirow{2}{*}{$\mathrm{P}=0.31$} \\
\hline & $\begin{array}{l}\text { Microscope } \\
\text { assisted }\end{array}$ & 43 & 9.06 & 3.40 & \\
\hline
\end{tabular}

\section{Discussion}

Myringoplasty is the surgical procedure to repair the tympanic membrane perforation. It is one of the commonest surgical procedures performed in the Otorhinolaryngology practice. The present study was undertaken with the objective to assess the operative time, post operative pain, graft uptake and audiological gain in the group of the patients undergoing the endoscope assisted and the microscope assisted myringoplasty. While microscope assisted myringoplasty has been the traditional method of performing myringoplasty but in the patients with tortuosity of the external auditory canal (EAC) and bony canal overhang hampers the view of the tympanic membrane remnant and other middle ear structures, it leads to frequent manipulation of head of the patient. In some cases despite manipulation of head deeper structures cannot be visualised, canalplasty becomes unavoidable in them. This increases the operative time. Endoscope assisted myringoplasty is relatively new technique of performing myringoplasty.

It has improved the visual exposure of hidden structures and deep recesses, obtaining a wide angle view and it also helps in performing minimally invasive surgery with greater healthy tissues preservation $[13,14]$. Endoscope can be easily negotiated through tortuos EAC, thus hidden structures can be seen easily and canalplasty possibly avoided. Endoscope is said to bring the surgeon's eye to its tip thus providing panoramic view and magnifying the image by just getting close to the structures. This obviates the need of frequently manipulating the head of the patient. Usami S et al. [15] and Tarabichi M [16] also made similar observations in two different studies. The middle ear structures which are difficult to be visualised with operating microscope e.g.: anterior epitympanum, Eustachian tube area, round window niche, 


\section{Global Journal of Otolaryngology}

facial recess and sinus tympani, can also be effectively visualised with angled endoscopes. The endoscope has advantage of being light weight and relatively smaller in size hence can be used for performing surgeries in the camps.

The biggest demerit of endoscope assisted ear surgery is that it is one handed technique. The surgeon holds the endoscope in one hand while performing the surgery and other hand is free to perform surgery. To operate at time of excessive bleeding is difficult because only one hand is free and the tip of the endoscope has to be cleaned repeatedly. This problem can be sorted by using the stand for endoscope, which stabilises the endoscope in desired position. In this way both the hands of the surgeon become free. But stand for the endoscope has other limitations and its usefulness in endoscope assisted surgery is debated. The demerit of using the stand for endoscope is that the focus of the endoscope has to be changed frequently to visualise the tympanic membrane remnant and middle ear structures and also its tip has to be cleaned repeatedly. It becomes extremely difficult to perform these functions after fixing the endoscope in the stand.

The microscope assisted technique has advantage of both the hands being free to perform the surgery. Thus procedure is easily performed in the microscope assisted technique. Tarabichi M [16] and Karhuketo TS et al. [17] also made similar observation. The endoscopes provide monocular vision due to which depth perception is lost. Therefore extra precaution has to be taken while positioning the graft and working close to the vital structures. The chlorhexidine gluconate and cetrimide solution (Savlon) is used for defogging of endoscopes, the safety of which on the middle ear mucosa is not established.

In our study the operative time in the transcanal endoscope assisted group of the patients was $49.76 \pm 3.18$ minutes whereas in the transcanal microscope assisted group of the patients it was $62.37 \pm 3.69$ minutes and Endaural/Postaural microscope assisted group of the patients it was $72.15 \pm 2.56$ minutes. On applying the one way analysis of variance (ANOVA) test, the difference in the operative time between different groups came out to be highly statistically significant with $p$ value of $P=<0.001$. Further on applying post hoc Turkey's Honest Significant Difference test the inter group difference between transcanal endoscope assisted, transcanal microscope assisted and Endaural/Postaural microscope assisted groups was found to be statistically significant between each two groups. Our result for operative time was in concurrence with the results of Patel J et al. [18] and not in concurrence with the results of Harugop AS et al. [10].

The post operative pain in the different group of patients was evaluated in our study. The patients were interviewed one day post operatively and they were asked to grade the post operative pain on the numerical rating scale, $0-10$. Each patient was assigned a definite number on the 0-10 scale, depending upon the severity of the post operative pain. In our study the post operative pain in the transcanal endoscope assisted group of the patients has a numerical value of $3.56 \pm 1.28$ whereas in the transcanal microscope assisted group of the patients it was $4.40 \pm 1.35$ and Endaural/Postaural microscope assisted group of the patients it was $5.40 \pm 1.54$. One way analysis of variance (ANOVA) test was used for calculating statistical significance between these groups. The difference was found to be highly statistically significant with $p$ value of $\mathrm{P}=<0.001$. Post hoc Turkey's Honest Significant Difference test was applied after ANOVA and statistically significant inter group difference was found in the severity of pain between transcanal endoscope assisted, transcanal microscope assisted and Endaural/Postaural microscope assisted. Our result was in concurrence with the results of Patel J et al. [18] and Raj A et al. [19].

In our study the post operative graft uptake was better in the group of the patients undergoing endoscope assisted myringoplasty as compared to the microscope assisted myringoplasty. The success of graft uptake was $88 \%$ in the endoscope assisted group and $84 \%$ in the microscope assisted group after one month of surgery, the difference being statistically insignificant with $p$ value of $\mathrm{P}=0.56$. Similarly the success of graft uptake at three months and six months after was better in the endoscope assisted group but the difference was not statistically significant with $p$ value of $\mathrm{P}=0.62$ and $\mathrm{P}=0.39$ at $3 \& 6$ months respectively. Our result was in concurrence with the result of Raj A et al. [19] and not in concurrence with the result of Harugop AS et al [10].

The postoperative mean audiological gain in the group of the patients undergoing endoscope assisted myringoplasty was $9.33+3.19 \mathrm{~dB}$ and in the microscope assisted group it was $8.83+3.43 \mathrm{~dB}$ after one months of the surgery. The difference between both the groups was not statistically significant with $p$ value of $\mathrm{P}=0.45$. The postoperative audiological improvement at 3 and 6 months post operatively had $p$ value of $\mathrm{P}=0.36$ and $\mathrm{P}=0.31$, with no statistically significant difference. Our result for audiological gain was in concurrence with the result of Raj A t al [19].

\section{Conclusion}

The endoscope assisted ear surgery has revolutionized the concept of minimally invasive ear surgeries. The endoscopes provides wide angle, panoramic view of the tympanic membrane remnant and other middle ear structures in the patients undergoing ear surgery. Moreover the image provided by the endoscope can be magnified by bringing the endoscope close to the structures. The endoscopes have other privilege of being easily negotiable through the external auditory canal and thus reduce the need for canalplasty to be performed. In the above mentioned ways the endoscope overcomes most of the shortcomings of the microscope.

We conclude that in our study the operative time and post operative pain was less in the group of the patients undergoing the endoscope assisted surgery as compared to the microscope assisted surgery, the difference between the groups being statistically significant. In our study the tympanic membrane graft uptake was better and post operative audiological gain was more in the group of patients undergoing the endoscope assisted myringoplasty than the microscope assisted myringoplasty although the differ- 
ence between the groups was not statistically significant. Hence this study shows that the endoscopes can be used to perform ear surgeries with less post operative morbidity and better results.

\section{Compliance with Ethical Standards}

- The authors declare that they have no conflict of interest.

- $\quad$ All procedures performed in the study involving human participants were in accordance with the ethical standards of the institutional and/or national research committee and with the 1964 Helsinki declaration and its later amendments or comparable ethical standards.

- Informed consent was obtained from all individual participants included in the study.

\section{References}

1. Schuknecht HF (1993) Pathology of the ear ( $\left.2^{\text {nd }} e d n\right)$, Lea and Febiger. Philadelphia, USA, pp. 191-253.

2. Glasscock ME, Shambaugh GE (2003) Surgery of the Ear (5 th edn), Pathology and clinical course of inflammatory diseases of the middle ear. 21: 428-429.

3. Buckingham RA (1992) Fascia and perichondrium atrophy in tympanoplasty and recurrent middle ear atelectasis. Ann Otol Rhinol Laryngol 101(9): 755-758.

4. Browning GG, Merchant SN, Kelly G. Chronic otitis media. In: Gleeson MD (Ed,). Scott-Brown's Otorhinolaryngology, $\left(7^{\text {th }}\right.$ edn), Head and Neck Surgery 3: 3425.

5. Chen B, Fry TL, Fischer ND (1979) Otoscopy and photography: a new method. Ann Otol Rhinol Laryngol 88(Pt 1): 771-773.

6. Migirov L, Shapira Y, Hoeowitz Z, Wolf M (2011) Exclusive endoscopic ear surgery for acquired cholesteatoma. Otol Neurotol 32(3): 433-436.
7. Marchioni D, Alicandri-Ciufelli M, Piccinini A, Genovese E, Monzani D, et al. (2011) Surgical anatomy of the transcanal endoscopic approach to the tympanic facial nerve. Laryngoscope 121(7): 1563-1571.

8. Adbel BF, El dine MB, El Sajid I, Bakry M (2002) Sinus tympani endoscopic anatomy. Otolaryngology Head Neck Surgery 127(3): 158162.

9. el Guindy A (1992) Endoscopic transcanal myringoplasty. J Laryngol Otol 106(6): 493-495.

10. Harugop AS, Mudhol RS, Godhi RA (2008) A comparative study of endoscope assisted and microscope assisted myringoplasty. Indian J Otolaryngol Head Neck Surg 60(4): 298-302.

11. Coskun BU, Cinar U, Seven H, Ugur S, Dadas B (2006) The effects of incision types in myringoplasty operations on cosmetics. Eur Arch Otorhinolaryngol 263(9): 820-822.

12. Fisch U (2008) Tympanoplasty, mastoidectomy and stapes surgery, $\left(2^{\text {nd }}\right.$ edn), Thieme, New York, USA, pp. 8-11.

13. Marchioni D, Villari D, Mattioli F, Alicandri-Ciufelli M, Piccinini A, et al. (2013) Endoscopic management of attic cholesteatoma: a singleinstitution experience. Otolaryngol Clin N Am 46(2): 201-209.

14. Badr-El-Dine M, James AL, Panetti G, Marchioni D, Presutti L, et al. (2013) Instrumentation and technologies in endoscopic ear surgery. Otolaryngol Clin N Am 46(2): 211-225.

15. Usami S, Iijima N, Fujita S, Takumi Y (2001) Endoscope-assisted Myringoplasty. Otorhinolaryngology 63(5): 287-290.

16. Tarabichi M (1999) Endoscopic Middle Ear Surgery. Ann Otol Rhinol Laryngol 108(1): 39-46.

17. Karhuketo TS, Ilomäki JH, Puhakka HJ (2001) Tympanoscope-Assisted Myringoplasty. ORL J Otorhinolaryngol Relat Spec 63(6): 353-358.

18. Patel J, Aiyer RG, Gajjar Y, Gupta R, Raval J, et al. (2015) Endoscopic tympanoplasty vs microscopic tympanoplasty in tubotympanic csom: a comparative study of 44 cases. Int J Res Med Sci 3(8): 1953-1957.

19. Raj A, Meher R (2001) Endoscopic transcanal myringoplasty-A study. Indian J Otolaryngol Head Neck Surgery 53(1): 47-49. 